\title{
Molecular Dynamics Simulation Study of Permeation of Molecules through Skin Lipid Bilayer
}

\author{
Rakesh Gupta, D B Sridhar and Beena Rai*
}

Physical Science Research Area, TCS Research

Tata Research Development and Design Centre, Tata Consultancy Services, 54B, Hadapsar Industrial Estate, Pune - 411013, INDIA

*Corresponding author beena.rai@tcs.com

Fax: $\quad 91-20-66086399$

Tel: $\quad 91-20-66086203$

\section{Supplementary information}

\section{S1. Initial configuration}

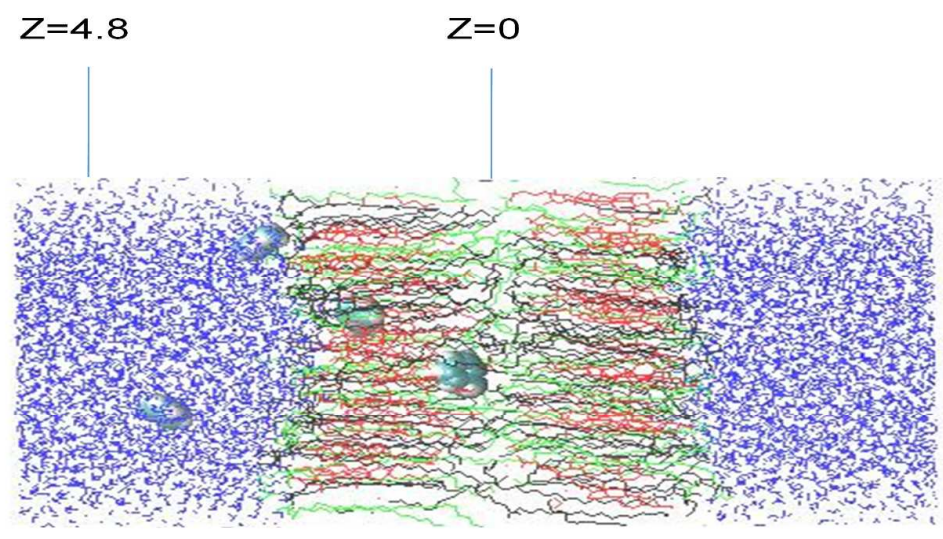

Figure S1. Snapshot of a system of four molecules constrained in single window. Z represents the bilayer normal.

TableS1: Distances between the constrained molecules and the bilayer center of mass $(\mathrm{z}=0)$ in each window. There are total 25 windows, each with 4 constrained molecules

\begin{tabular}{|c|c|c|c|c|c|c|c|c|c|c|c|c|c|c|c|c|c|c|c|c|c|c|c|c|c|}
\hline $\begin{array}{c}\text { Window } \\
\text { no } \rightarrow \\
\text { Solute no } \\
\downarrow\end{array}$ & 1 & 2 & 3 & 4 & 5 & 6 & 7 & 8 & 9 & 10 & 11 & 12 & 13 & 14 & 15 & 16 & 17 & 18 & 19 & 20 & 21 & 22 & 23 & 24 & 25 \\
\hline 1 & 4.8 & 4.6 & 4.4 & 4.2 & 4.0 & 3.8 & 3.6 & 3.4 & 3.2 & 3.0 & 2.8 & 2.6 & 2.4 & 2.2 & 2.0 & 1.8 & 1.6 & 1.4 & 1.2 & 1.0 & 0.8 & 0.6 & 0.4 & 0.2 & 0.0 \\
\hline 2 & 3.6 & 3.4 & 3.2 & 3.0 & 2.8 & 2.6 & 2.4 & 2.2 & 2.0 & 1.8 & 1.6 & 1.4 & 1.2 & 1.0 & 0.8 & 0.6 & 0.4 & 0.2 & 0.0 & 4.8 & 4.6 & 4.4 & 4.2 & 4.0 & 3.8 \\
\hline 3 & 2.4 & 2.2 & 2.0 & 1.8 & 1.6 & 1.4 & 1.2 & 1.0 & 0.8 & 0.6 & 0.4 & 0.2 & 0.0 & 4.8 & 4.6 & 4.4 & 4.2 & 4.0 & 3.8 & 3.6 & 3.4 & 3.2 & 3.0 & 2.8 & 2.6 \\
\hline 4 & 1.2 & 1.0 & 0.8 & 0.6 & 0.4 & 0.2 & 0.0 & 4.8 & 4.6 & 4.4 & 4.2 & 4.0 & 3.8 & 3.6 & 3.4 & 3.2 & 3.0 & 2.8 & 2.6 & 2.4 & 2.2 & 2.0 & 1.8 & 1.6 & 1.4 \\
\hline
\end{tabular}


S2. Effect of sampling time on free energy profile.
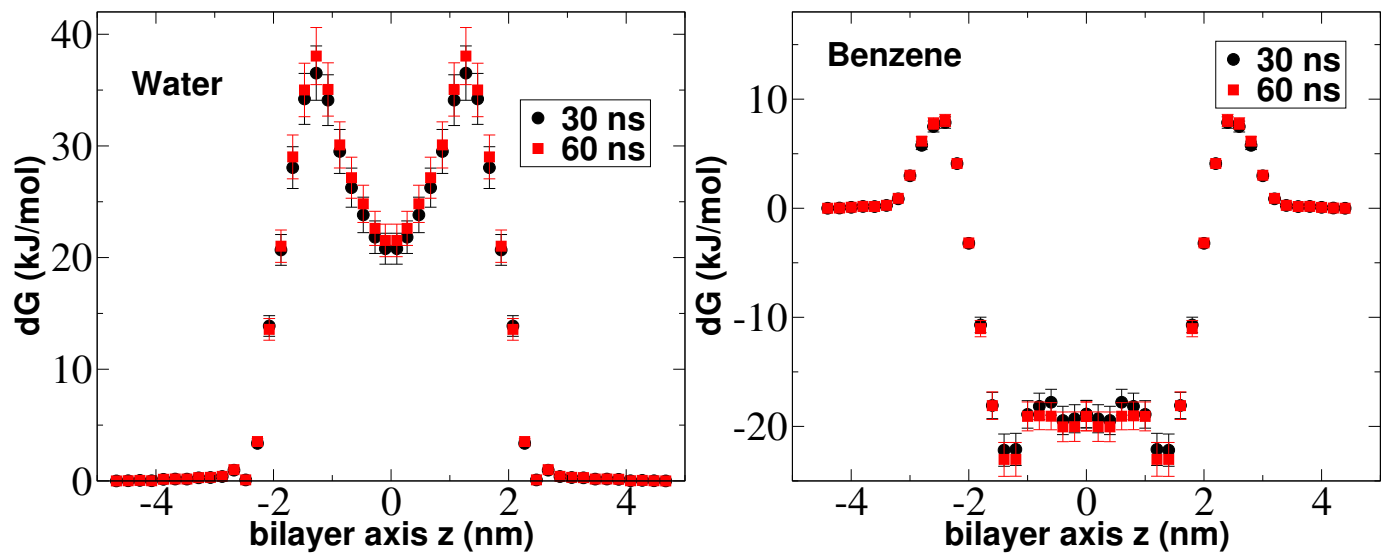

Figure S2. Effect of sampling time on the free energy of permeation of water and benzene.

\section{S3. Force field parameters}

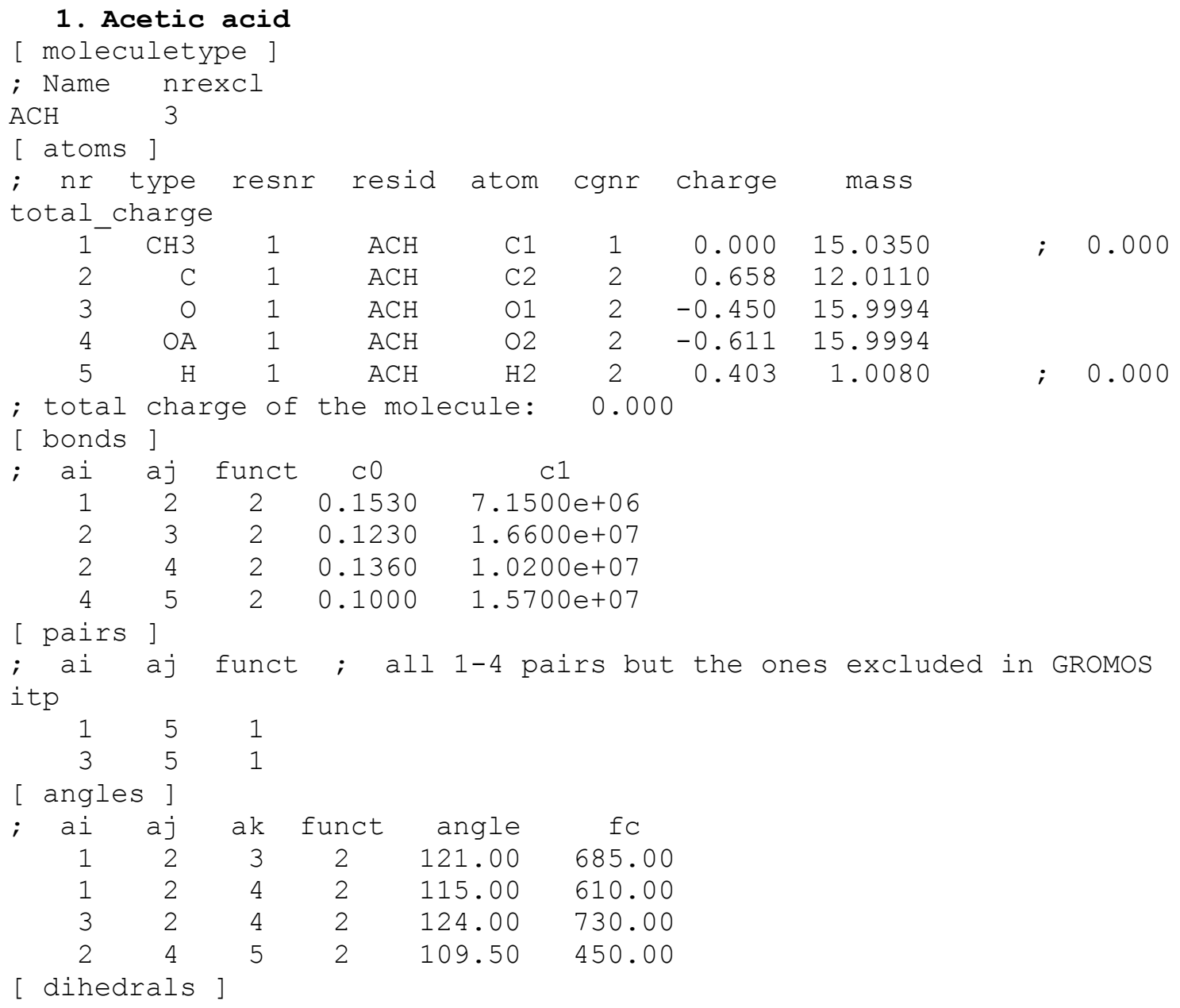




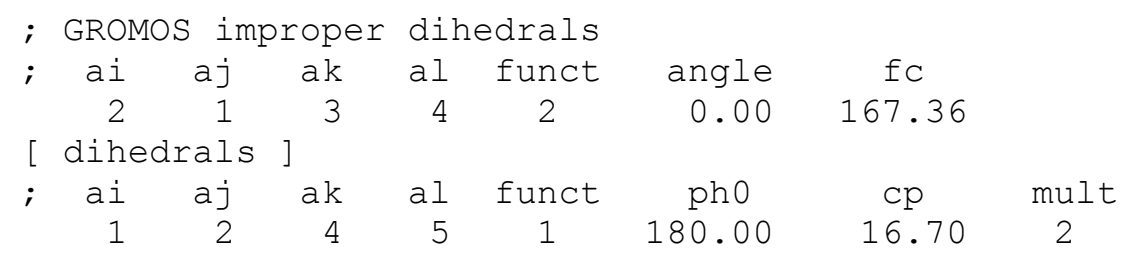

\section{Benzne}



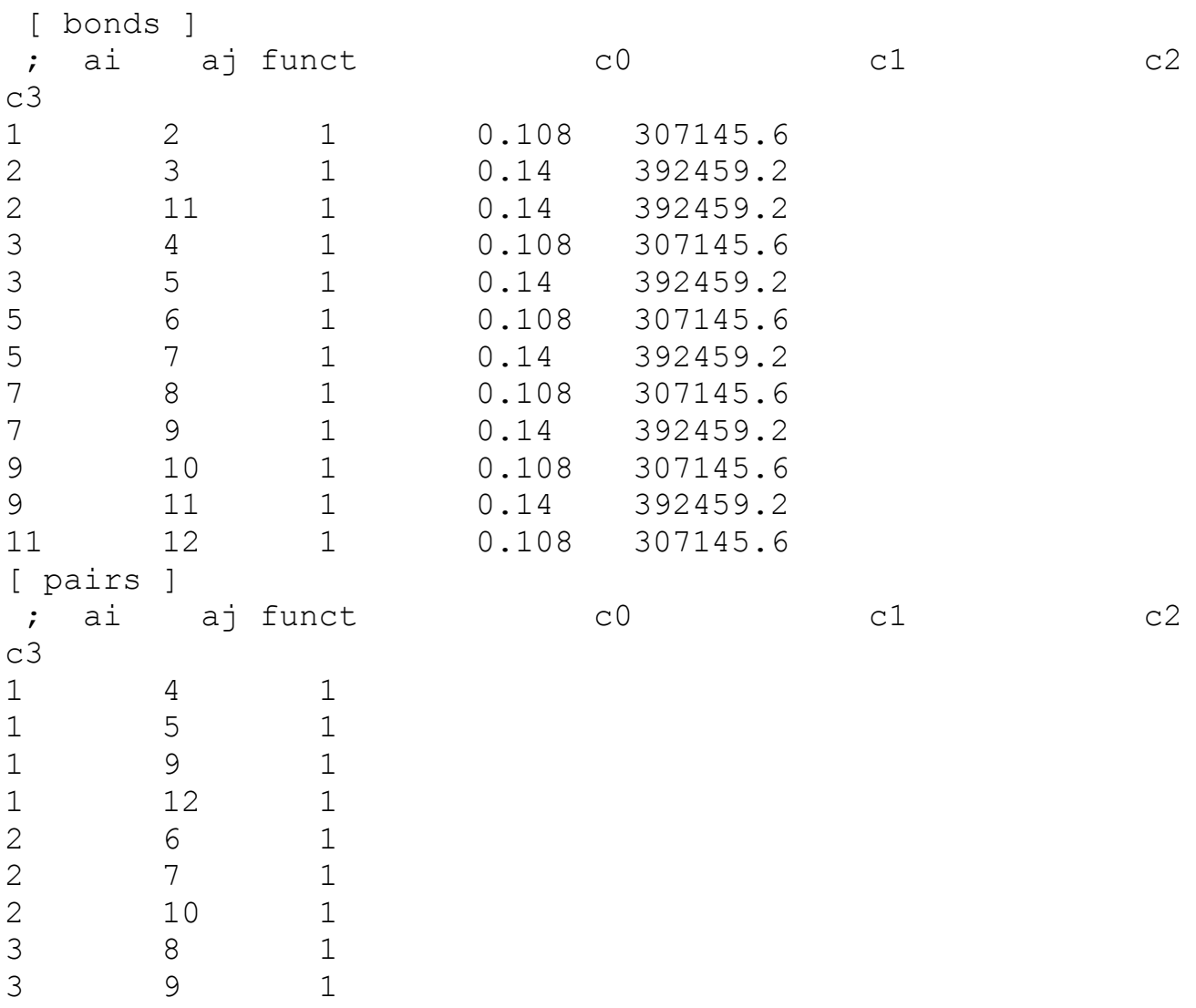




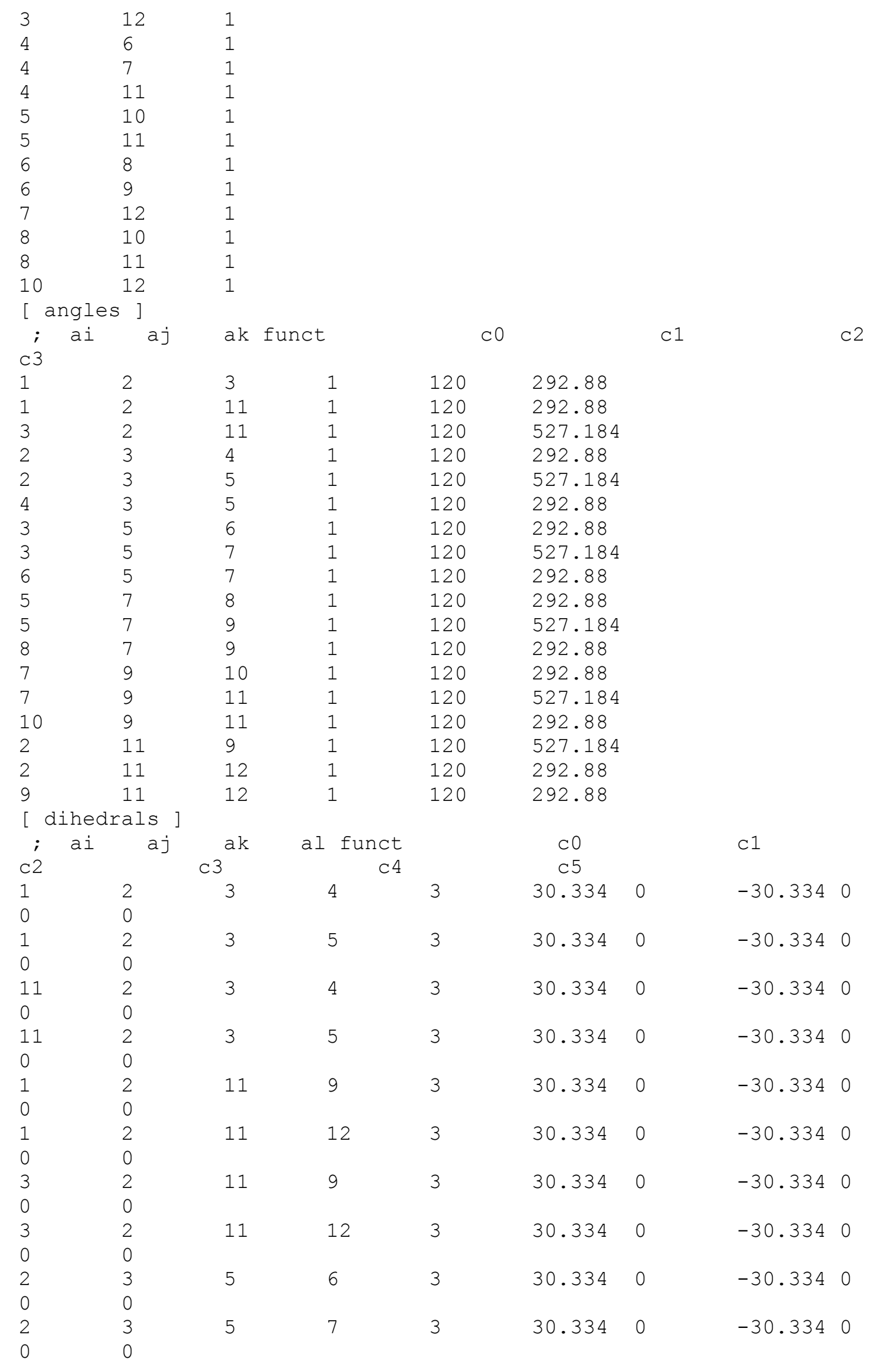




\begin{tabular}{|c|c|c|c|c|c|c|c|}
\hline 4 & 3 & 5 & 6 & 3 & 30.334 & 0 & -30.334 \\
\hline 0 & 0 & & & & & & \\
\hline 4 & 3 & 5 & 7 & 3 & 30.334 & 0 & -30.334 \\
\hline 0 & 0 & & & & & & \\
\hline 3 & 5 & 7 & 8 & 3 & 30.334 & 0 & -30.334 \\
\hline 0 & 0 & & & & & & \\
\hline 3 & 5 & 7 & 9 & 3 & 30.334 & 0 & -30.334 \\
\hline 0 & 0 & & & & & & \\
\hline 6 & 5 & 7 & 8 & 3 & 30.334 & 0 & -30.334 \\
\hline 0 & 0 & & & & & & \\
\hline 6 & 5 & 7 & 9 & 3 & 30.334 & 0 & -30.334 \\
\hline 0 & 0 & & & & & & \\
\hline 5 & 7 & 9 & 10 & 3 & 30.334 & 0 & -30.334 \\
\hline 0 & 0 & & & & & & \\
\hline 5 & 7 & 9 & 11 & 3 & 30.334 & 0 & -30.334 \\
\hline 0 & 0 & & & & & & \\
\hline 8 & 7 & 9 & 10 & 3 & 30.334 & 0 & -30.334 \\
\hline 0 & 0 & & & & & & \\
\hline 8 & 7 & 9 & 11 & 3 & 30.334 & 0 & -30.334 \\
\hline 0 & 0 & & & & & & \\
\hline 7 & 9 & 11 & 2 & 3 & 30.334 & 0 & -30.334 \\
\hline 0 & 0 & & & & & & \\
\hline 7 & 9 & 11 & 12 & 3 & 30.334 & 0 & -30.334 \\
\hline 0 & 0 & & & & & & \\
\hline 10 & 9 & 11 & 2 & 3 & 30.334 & 0 & -30.334 \\
\hline 0 & 0 & & & & & & \\
\hline 10 & 9 & 11 & 12 & 3 & 30.334 & 0 & -30.334 \\
\hline 0 & 0 & & & & & & \\
\hline 2 & 11 & 3 & 1 & 1 & 180 & 4.6 & 2 \\
\hline 3 & 2 & 5 & 4 & 1 & 180 & 4.6 & 2 \\
\hline 5 & 3 & 7 & 6 & 1 & 180 & 4.6 & 2 \\
\hline 7 & 5 & 9 & 8 & 1 & 180 & 4.6 & 2 \\
\hline 9 & 7 & 11 & 10 & 1 & 180 & 4.6 & 2 \\
\hline 11 & 9 & 2 & 12 & 1 & 180 & 4.6 & 2 \\
\hline 2 & 3 & 5 & 7 & 1 & 180 & 4.6 & 2 \\
\hline 3 & 5 & 7 & 9 & 1 & 180 & 4.6 & 2 \\
\hline 5 & 7 & 9 & 11 & 1 & 180 & 4.6 & 2 \\
\hline 7 & 9 & 11 & 2 & 1 & 180 & 4.6 & 2 \\
\hline 9 & 11 & 2 & 3 & 1 & 180 & 4.6 & 2 \\
\hline 11 & 2 & 3 & 5 & 1 & 180 & 4.6 & 2 \\
\hline
\end{tabular}

\section{3. butanol}

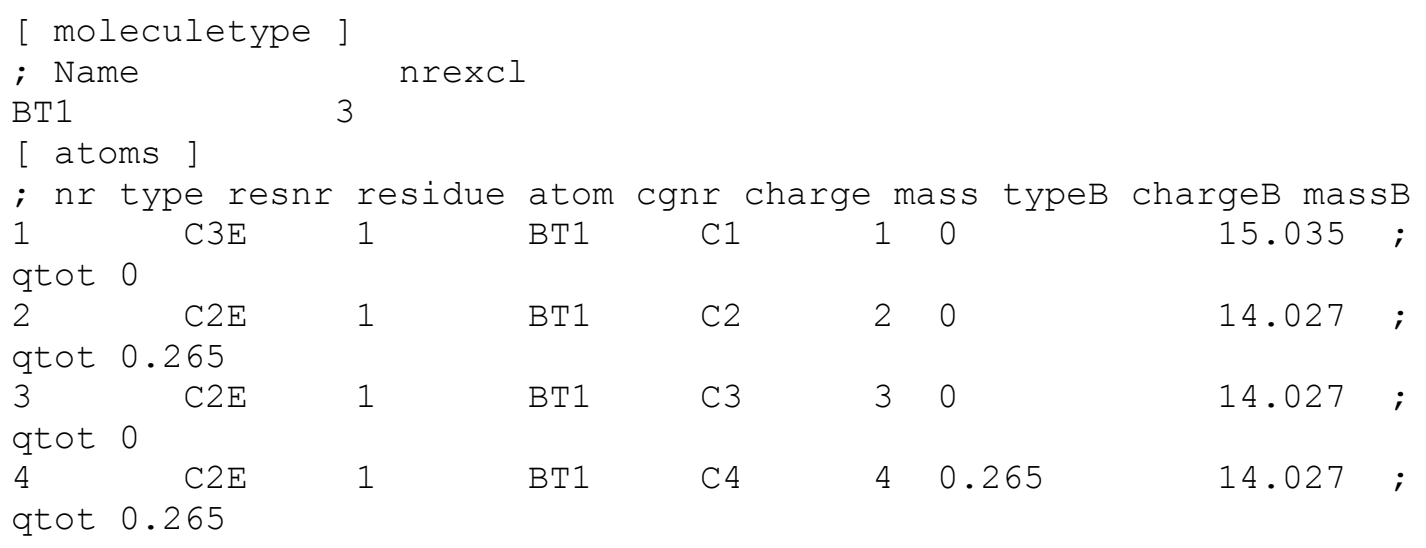




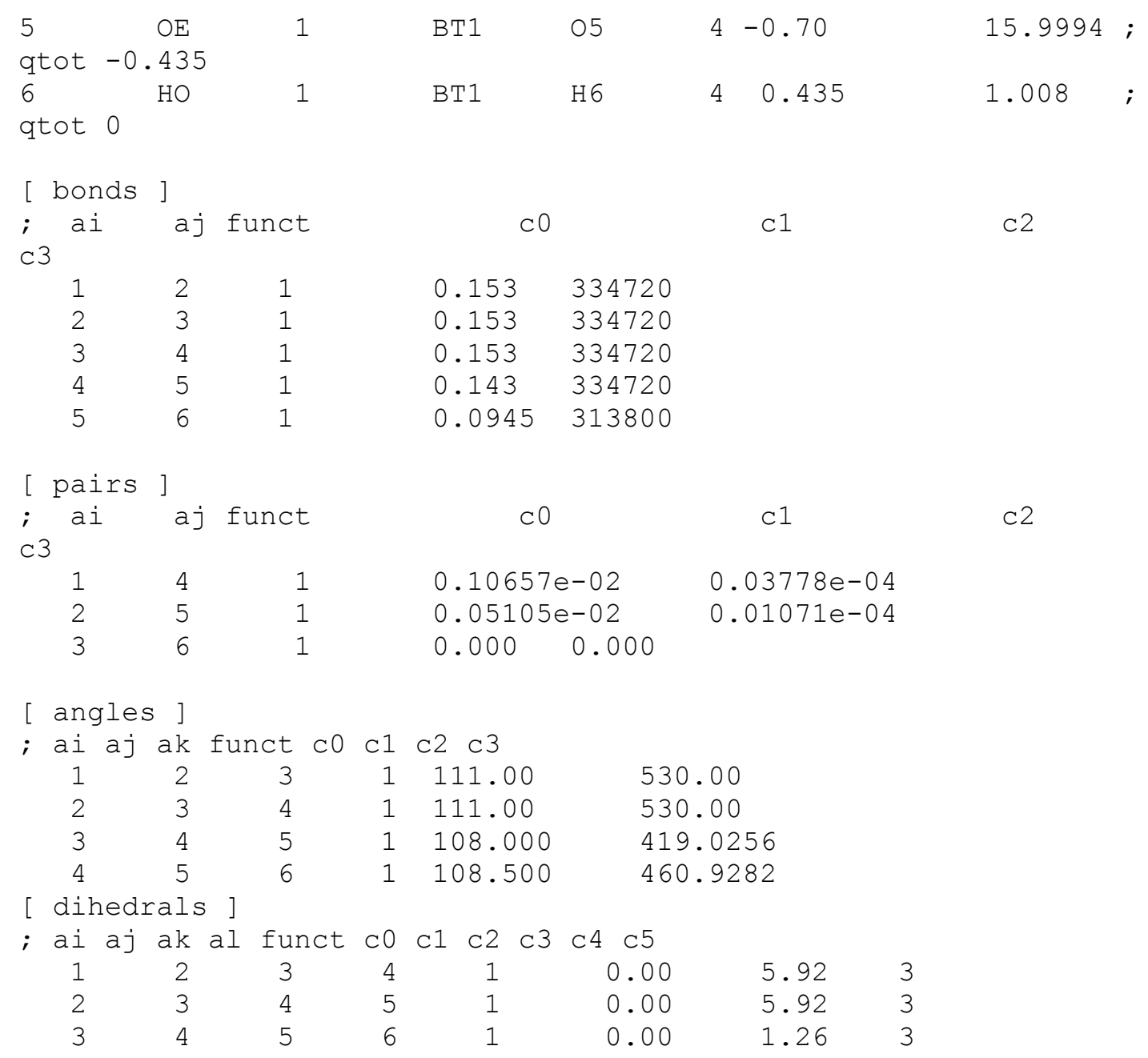

\section{DMSO}

[ moleculetype ]

; name nrexcl

DM1 2

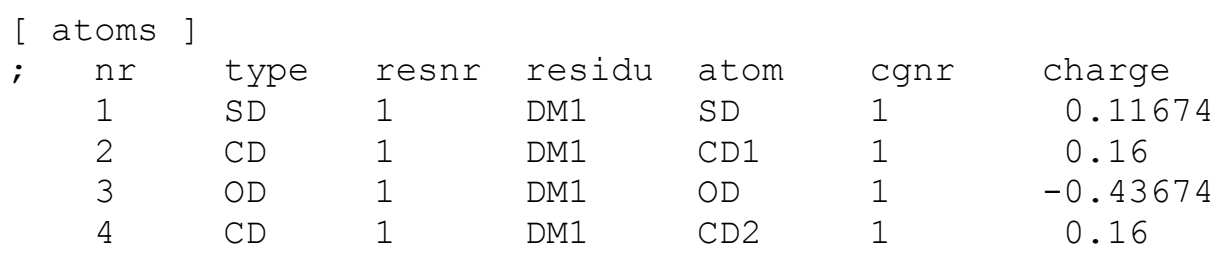

[ bonds ]

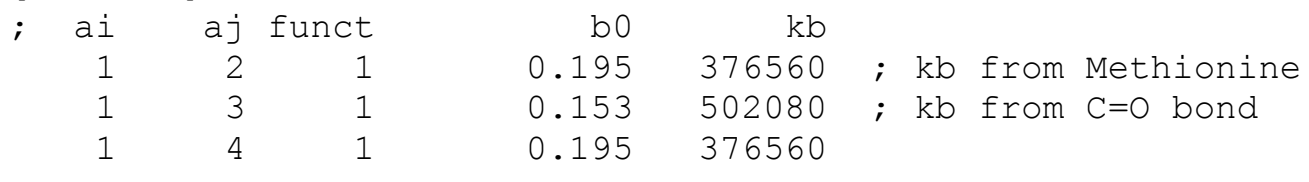

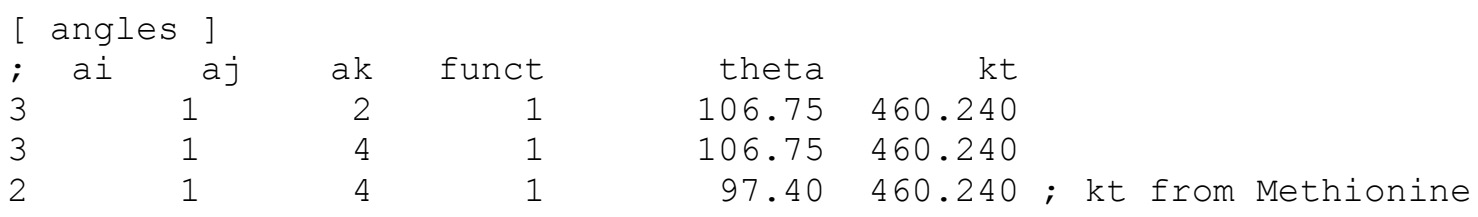




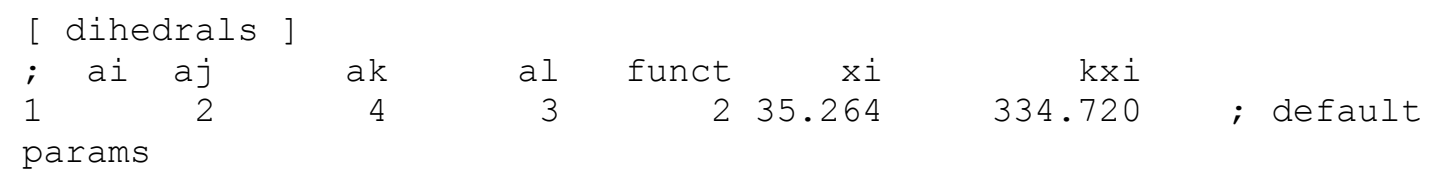

\section{5. ethanol}

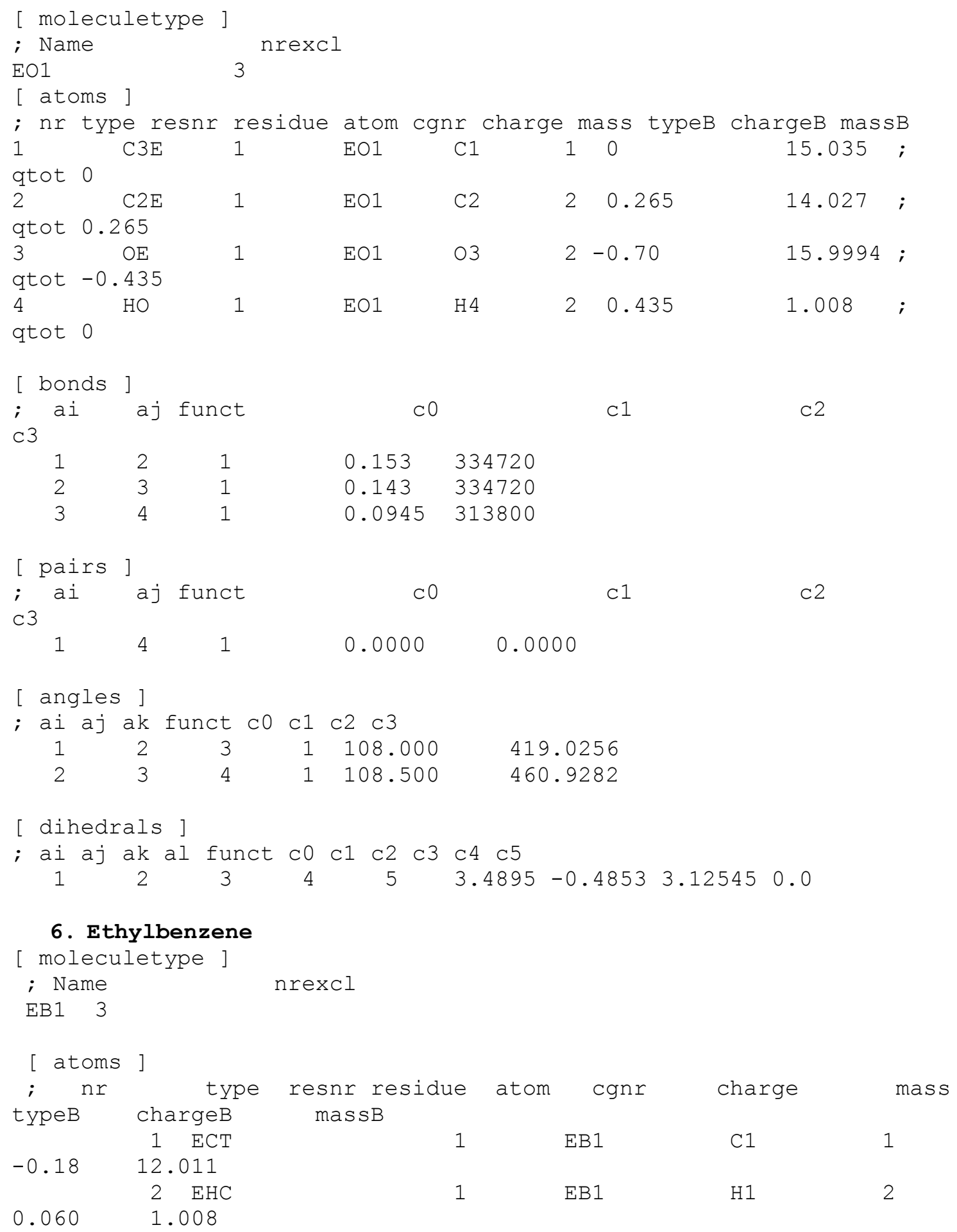




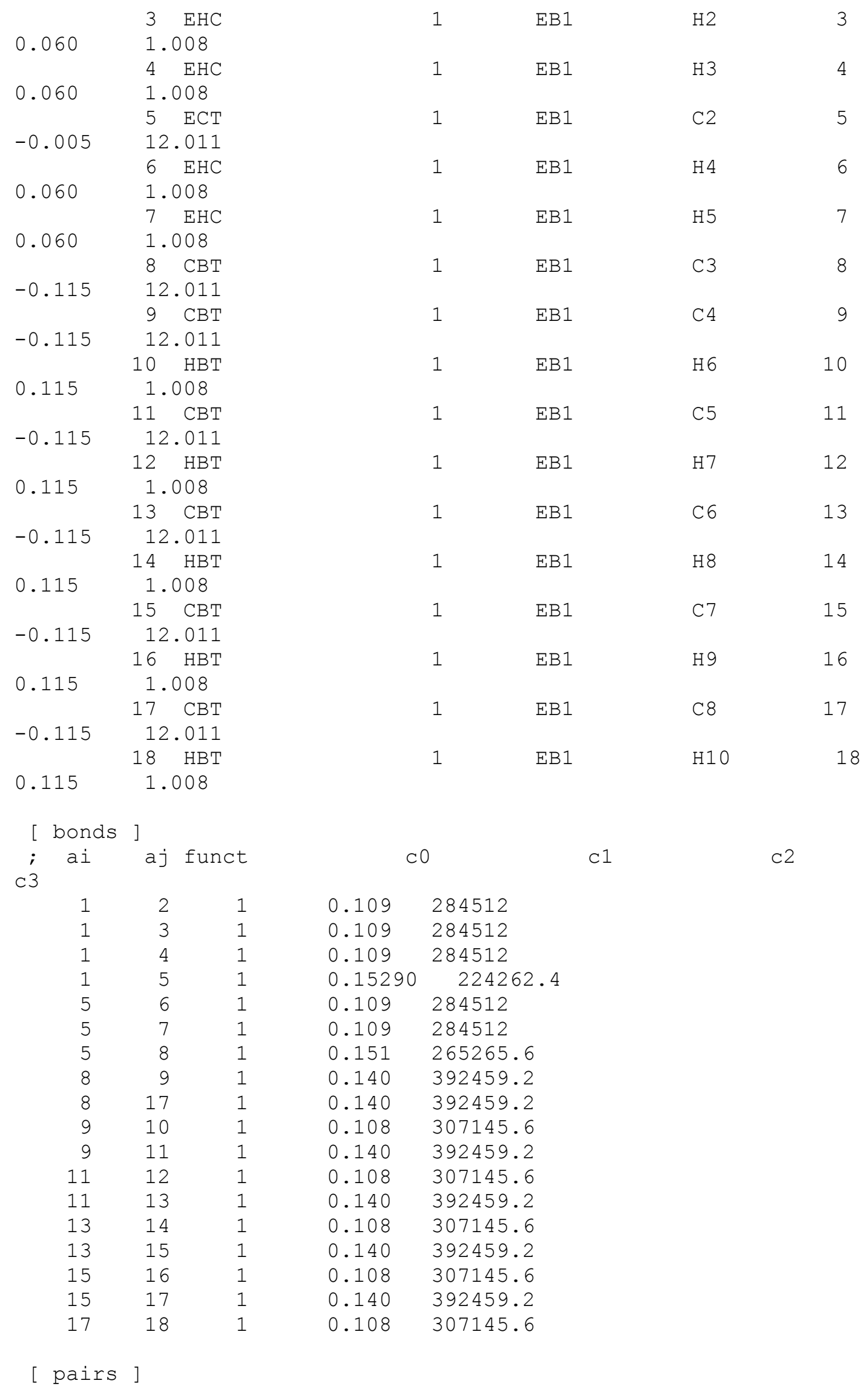




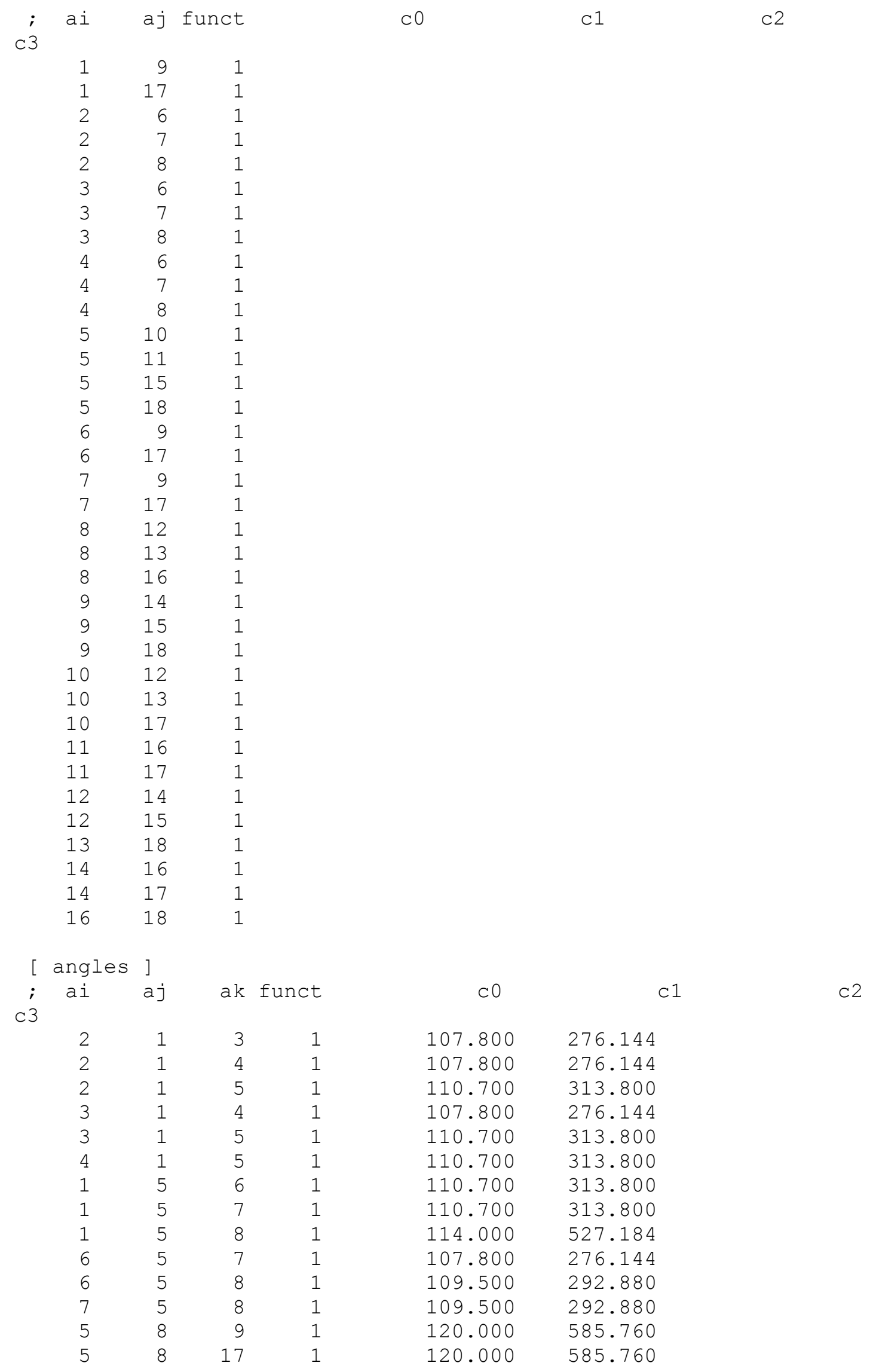




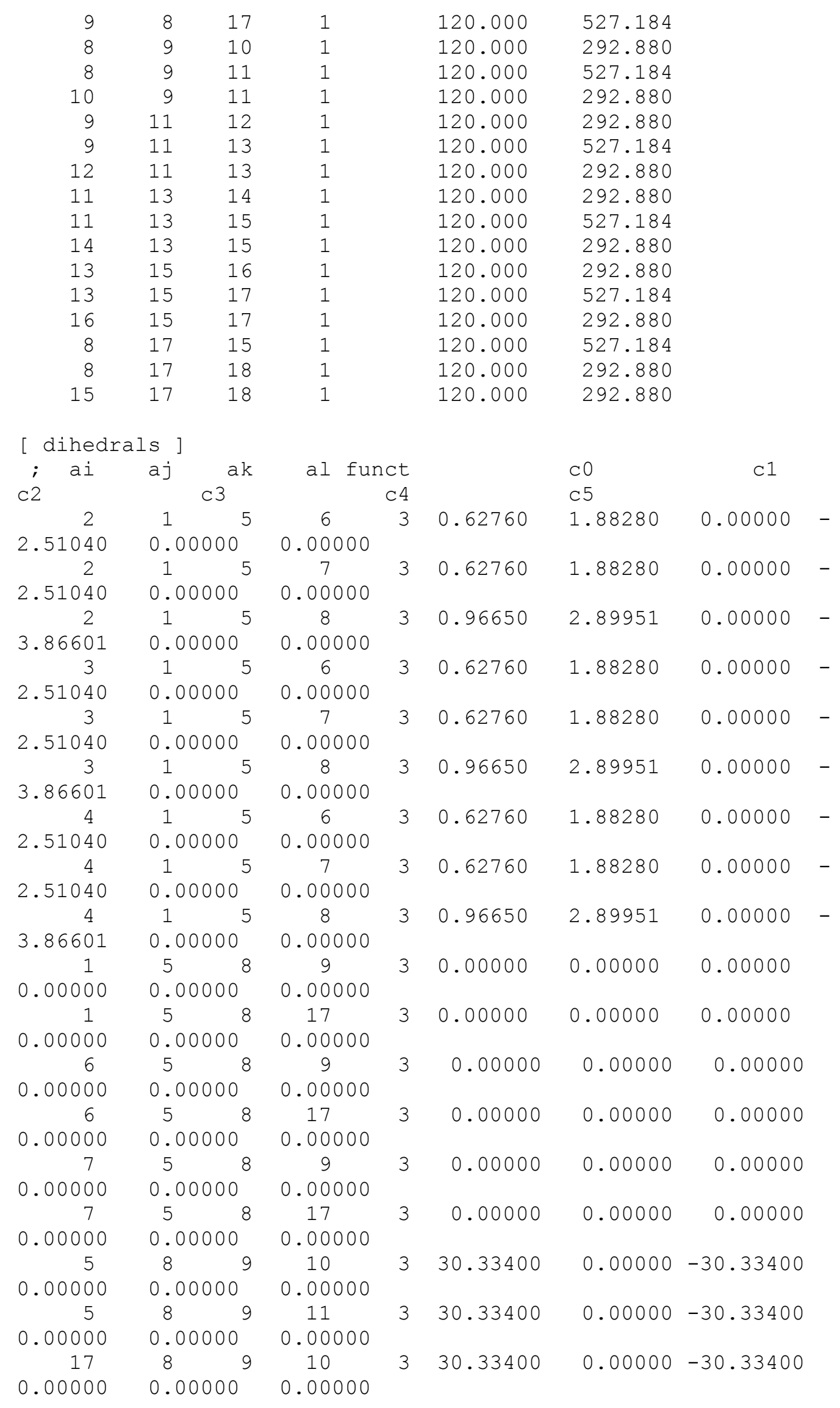




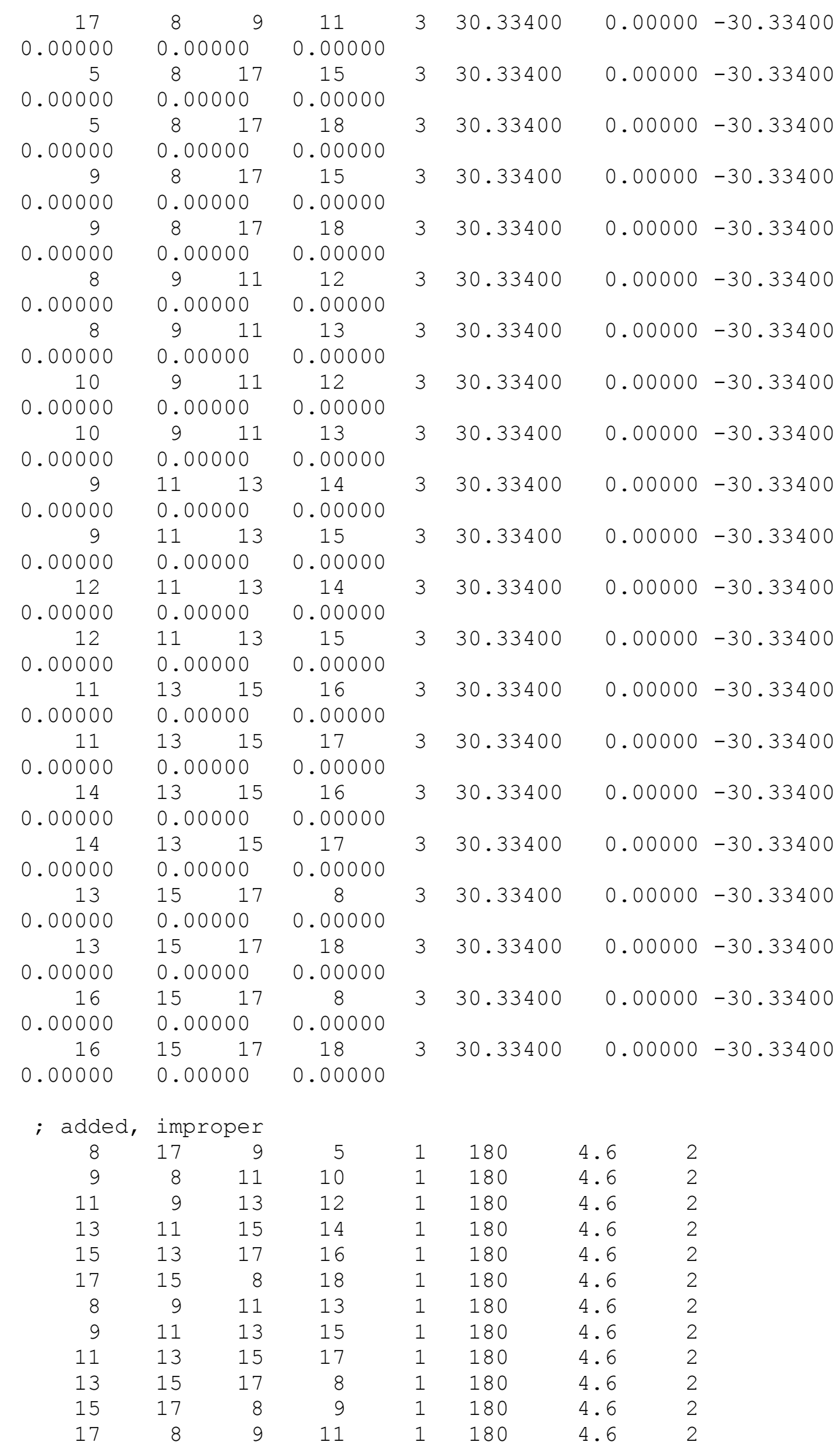




\section{Oxygen}

; [atomtypes]

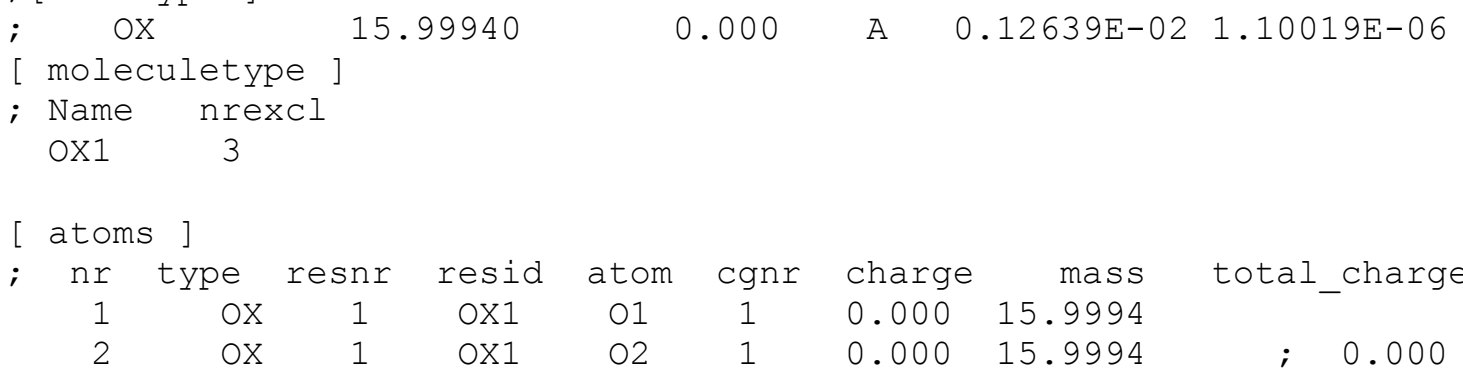

[ bonds ]

; ai aj funct c0 c1

$\begin{array}{lllll}1 & 2 & 1 & 0.1016 & 502080 .\end{array}$

\section{Phenol}

[ moleculetype ]

; Name

nrexcl

PH1 3

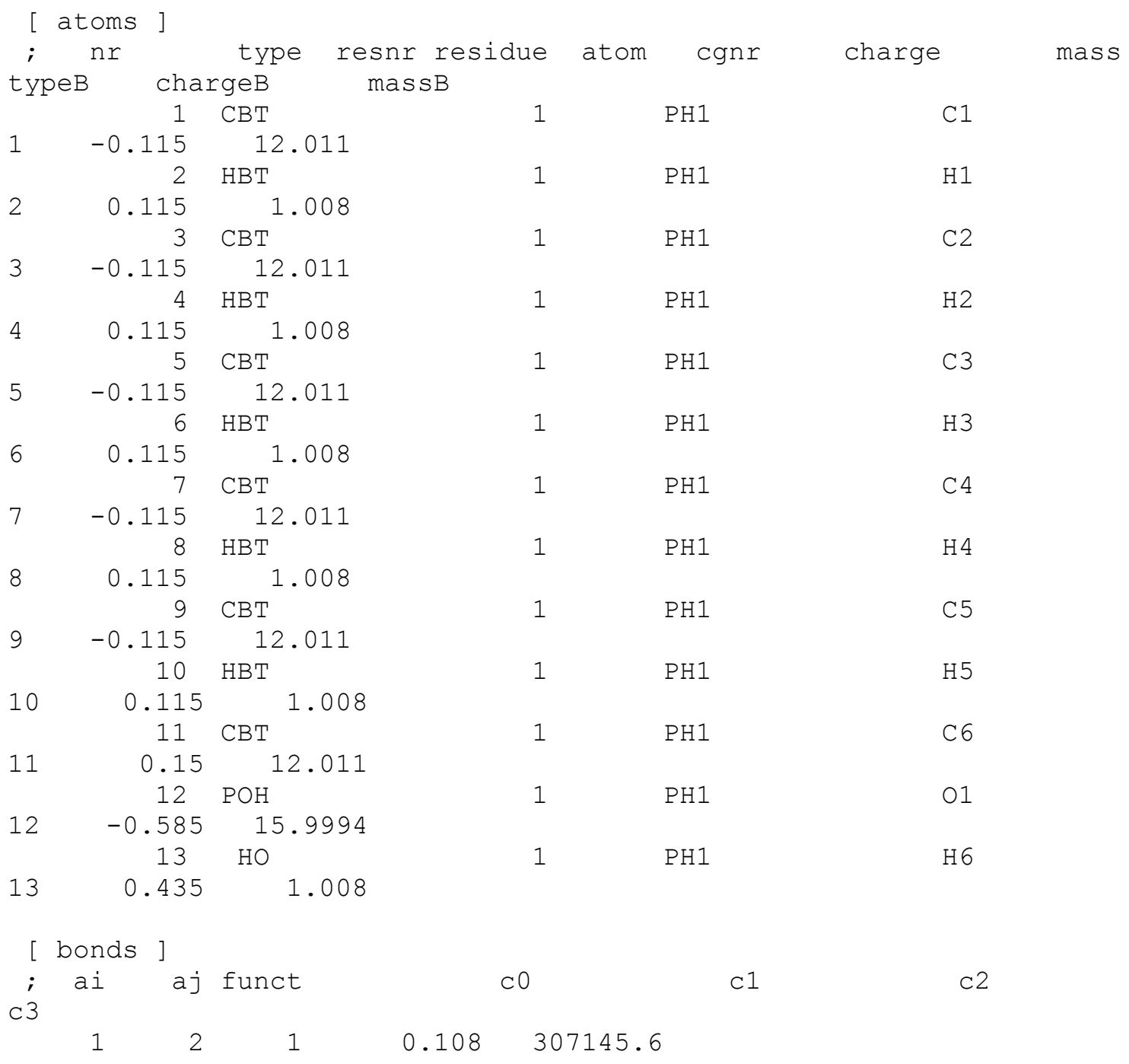




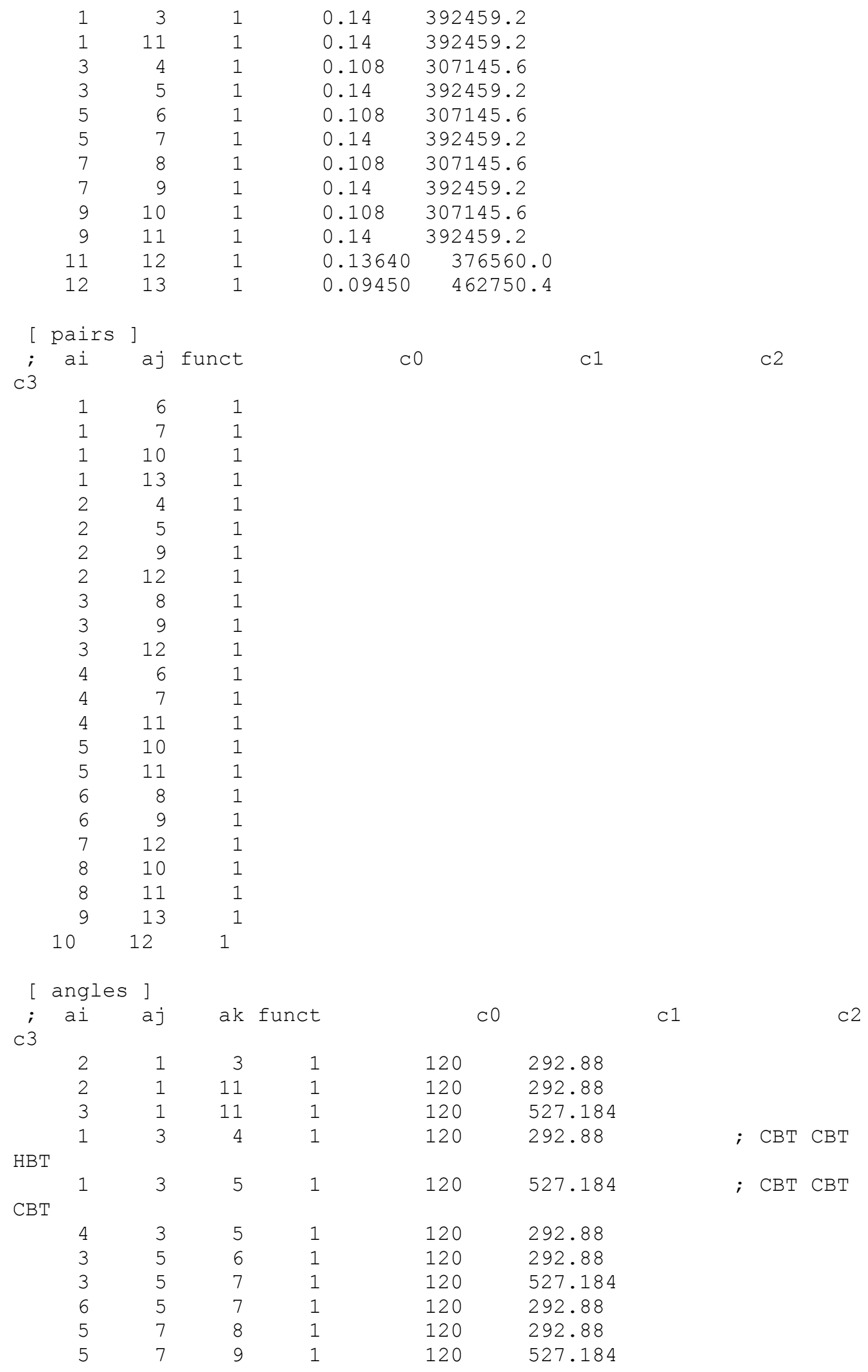




\begin{tabular}{|c|c|c|c|c|c|c|c|c|c|c|}
\hline & 8 & 7 & 9 & 1 & & 120 & \multicolumn{4}{|l|}{292.88} \\
\hline & 7 & 9 & 10 & 1 & & 120 & \multicolumn{4}{|l|}{292.88} \\
\hline & 7 & 9 & 11 & 1 & & 120 & \multicolumn{4}{|l|}{527.184} \\
\hline & 10 & 9 & 11 & 1 & & 120 & \multicolumn{4}{|l|}{292.88} \\
\hline & 1 & 11 & 9 & 1 & & 120 & \multicolumn{4}{|l|}{527.184} \\
\hline & 1 & 11 & 12 & 1 & & 120.000 & \multicolumn{4}{|c|}{585.760} \\
\hline & 9 & 11 & 12 & 1 & & 120.000 & \multicolumn{4}{|c|}{585.760} \\
\hline & 11 & 12 & 13 & 1 & & 113.000 & \multicolumn{4}{|c|}{292.880} \\
\hline \multicolumn{11}{|c|}{ [ dihedrals ] } \\
\hline ; & ai & \multirow{2}{*}{\multicolumn{2}{|c|}{$c 3^{a k}$}} & al & \multirow{2}{*}{\multicolumn{2}{|c|}{$\begin{array}{r}\text { funct } \\
\text { c4 }\end{array}$}} & \multirow{2}{*}{\multicolumn{2}{|c|}{$\mathrm{C} 0$}} & \\
\hline \multirow[t]{2}{*}{ c2 } & & & & & & & & & \multicolumn{2}{|l|}{ c1 } \\
\hline & 2 & 1 & 3 & 4 & 3 & & 30.334 & 0 & -30.334 & \\
\hline \multirow[t]{2}{*}{0} & & & & & & & & & & \\
\hline & 2 & 1 & 3 & 5 & 3 & & 30.334 & 0 & -30.334 & \\
\hline 0 & & & & & & & & & & \\
\hline & 11 & 1 & 3 & 4 & 3 & & 30.334 & 0 & -30.334 & 0 \\
\hline 0 & & & & & & & & & & \\
\hline & 11 & 1 & 3 & 5 & 3 & & 30.334 & 0 & -30.334 & 0 \\
\hline 0 & & & & & & & & & & \\
\hline & 2 & 1 & 11 & 9 & 3 & & 30.334 & 0 & -30.334 & 0 \\
\hline 0 & & & & & & & & & & \\
\hline & 2 & 1 & 11 & 12 & 3 & & 30.334 & 0 & -30.334 & 0 \\
\hline 0 & & & & & & & & & & \\
\hline & 3 & 1 & 11 & 9 & 3 & & 30.334 & 0 & -30.334 & 0 \\
\hline 0 & & & & & & & & & & \\
\hline & 3 & 1 & 11 & 12 & 3 & & 30.334 & 0 & -30.334 & 0 \\
\hline 0 & & & & & & & & & & \\
\hline & 1 & 3 & 5 & 6 & 3 & & 30.334 & 0 & -30.334 & 0 \\
\hline 0 & & & & & & & & & & \\
\hline & 1 & 3 & 5 & 7 & 3 & & 30.334 & 0 & -30.334 & 0 \\
\hline 0 & & & & & & & & & & \\
\hline & 1 & 3 & 5 & 7 & 3 & & 30.334 & 0 & -30.334 & 0 \\
\hline 0 & & & & & & & & & & \\
\hline & 4 & 3 & 5 & 6 & 3 & & 30.334 & 0 & -30.334 & 0 \\
\hline 0 & & & & & & & & & & \\
\hline & 4 & 3 & 5 & 7 & 3 & & 30.334 & 0 & -30.334 & 0 \\
\hline 0 & & & & & & & & & & \\
\hline & 3 & 5 & 7 & 8 & 3 & & 30.334 & 0 & -30.334 & 0 \\
\hline 0 & & & & & & & & & & \\
\hline & 3 & 5 & 7 & 9 & 3 & & 30.334 & 0 & -30.334 & 0 \\
\hline 0 & & & & & & & & & & \\
\hline & 6 & 5 & 7 & 8 & 3 & & 30.334 & 0 & -30.334 & 0 \\
\hline 0 & & & & & & & & & & \\
\hline & 6 & 5 & 7 & 9 & 3 & & 30.334 & 0 & -30.334 & 0 \\
\hline 0 & & & & & & & & & & \\
\hline & 5 & 7 & 9 & 10 & 3 & & 30.334 & 0 & -30.334 & 0 \\
\hline 0 & & & & & & & & & & \\
\hline & 5 & 7 & 9 & 11 & 3 & & 30.334 & 0 & -30.334 & 0 \\
\hline 0 & & & & & & & & & & \\
\hline & 8 & 7 & 9 & 10 & 3 & & 30.334 & 0 & -30.334 & 0 \\
\hline 0 & & & & & & & & & & \\
\hline & 8 & 7 & 9 & 11 & 3 & & 30.334 & 0 & -30.334 & 0 \\
\hline 0 & & & & & & & & & & \\
\hline & 7 & 9 & 11 & 1 & 3 & & 30.334 & 0 & -30.334 & 0 \\
\hline
\end{tabular}




\begin{tabular}{|c|c|c|c|c|c|c|c|c|}
\hline \multirow[b]{2}{*}{0} & \multirow[t]{2}{*}{9} & \multirow[t]{2}{*}{11} & \multirow[t]{2}{*}{12} & \multicolumn{2}{|l|}{3} & \multirow[t]{2}{*}{30.334} & \multirow[t]{2}{*}{0} & \multirow[t]{2}{*}{-30.334} \\
\hline & & & & & & & & \\
\hline 10 & 9 & 11 & 1 & 3 & & 30.334 & 0 & -30.334 \\
\hline 0 & & & & & & & & \\
\hline 10 & 9 & 11 & 12 & 3 & & 30.334 & 0 & -30.334 \\
\hline 0 & & & & & & & & \\
\hline 1 & 11 & 12 & 13 & 3 & & 7.0374 & 0 & -7.0374 \\
\hline 0 & & & & & & & & \\
\hline 9 & 11 & 12 & 13 & 3 & & 7.0374 & 0 & -7.0374 \\
\hline 0 & & & & & & & & \\
\hline ; add & $i m p$ & per & & & & & & \\
\hline 1 & 11 & 3 & 2 & 1 & 180 & 4.6 & 2 & \\
\hline 3 & 1 & 5 & 4 & 1 & 180 & 4.6 & 2 & \\
\hline 5 & 3 & 7 & 6 & 1 & 180 & 4.6 & 2 & \\
\hline 7 & 5 & 9 & 8 & 1 & 180 & 4.6 & 2 & \\
\hline 9 & 7 & 11 & 10 & 1 & 180 & 4.6 & 2 & \\
\hline 11 & 9 & 1 & 12 & 1 & 180 & 4.6 & 2 & \\
\hline 1 & 3 & 5 & 7 & 1 & 180 & 4.6 & 2 & \\
\hline 3 & 5 & 7 & 9 & 1 & 180 & 4.6 & 2 & \\
\hline 5 & 7 & 9 & 11 & 1 & 180 & 4.6 & 2 & \\
\hline 7 & 9 & 11 & 1 & 1 & 180 & 4.6 & 2 & \\
\hline 9 & 11 & 1 & 3 & 1 & 180 & 4.6 & 2 & \\
\hline 11 & 1 & 3 & 5 & 1 & 180 & 4.6 & 2 & \\
\hline
\end{tabular}

\section{Styrene}

[ moleculetype ]

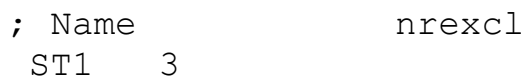

nrexcl

ST1 3

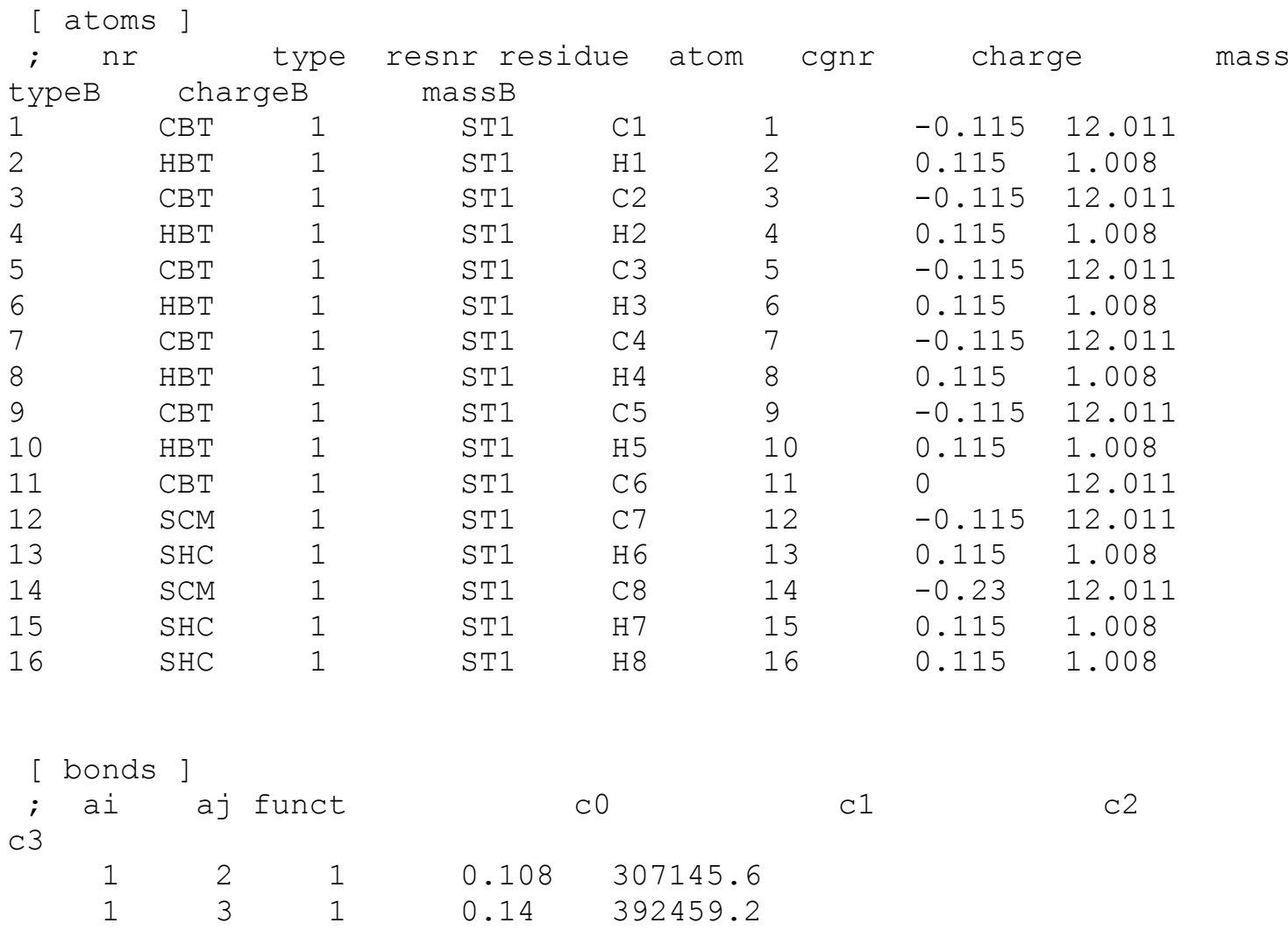




$$
\begin{aligned}
& \begin{array}{rrrlr}
1 & 11 & 1 & 0.14 & 392459.2 \\
3 & 4 & 1 & 0.108 & 307145.6 \\
3 & 5 & 1 & 0.14 & 392459.2 \\
5 & 6 & 1 & 0.108 & 307145.6 \\
5 & 7 & 1 & 0.14 & 392459.2 \\
7 & 8 & 1 & 0.108 & 307145.6 \\
7 & 9 & 1 & 0.14 & 392459.2 \\
9 & 10 & 1 & 0.108 & 307145.6 \\
9 & 11 & 1 & 0.14 & 392459.2 \\
11 & 12 & 1 & 0.14330 & 357313.6 \\
12 & 13 & 1 & 0.10800 & 284512.0 \\
12 & 14 & 1 & 0.13400 & 459403.2 \\
14 & 15 & 1 & 0.10800 & 284512.0 \\
14 & 16 & 1 & 0.10800 & 284512.0
\end{array} \\
& \text { [ pairs ] } \\
& \text {; ai aj funct }
\end{aligned}
$$




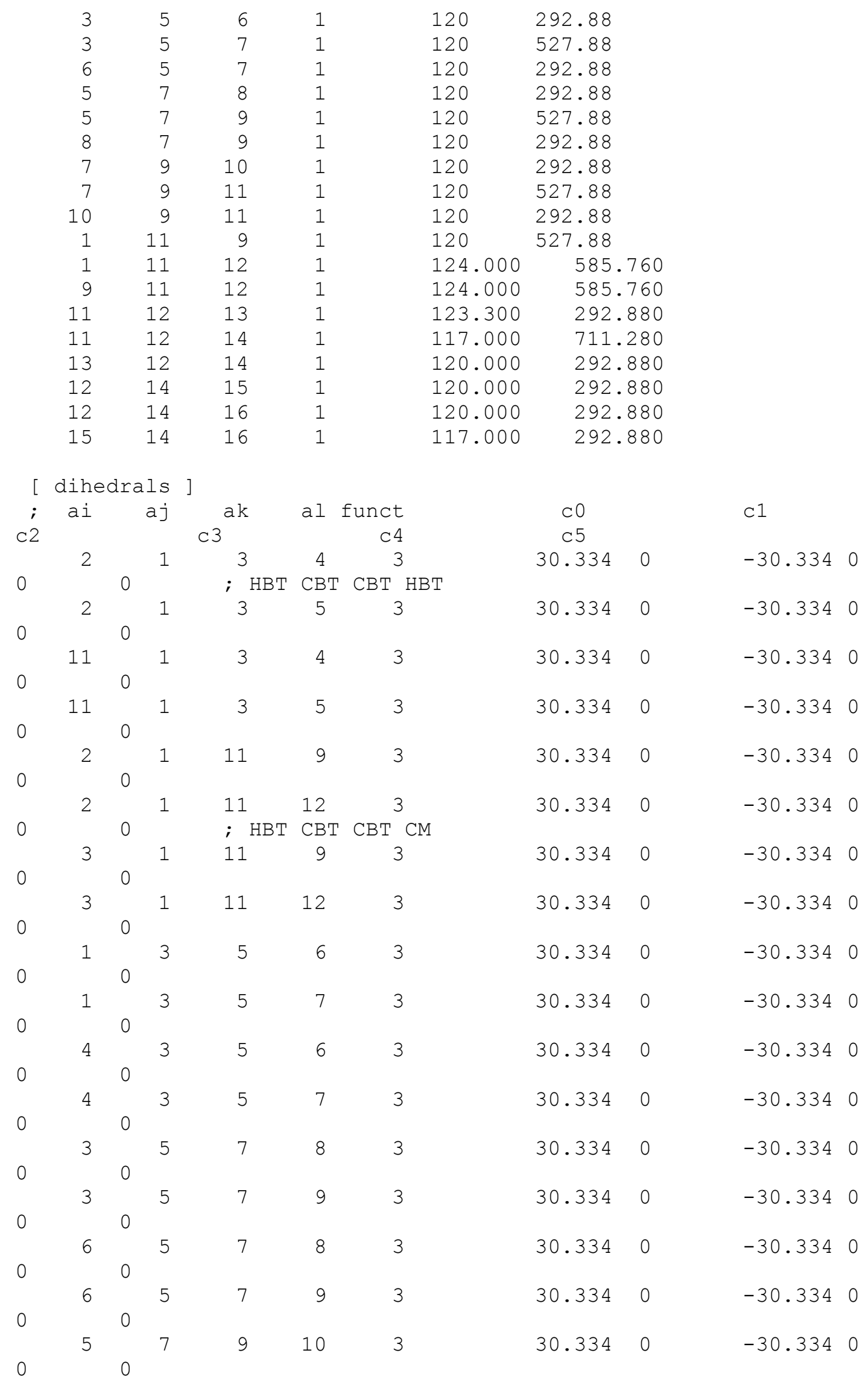




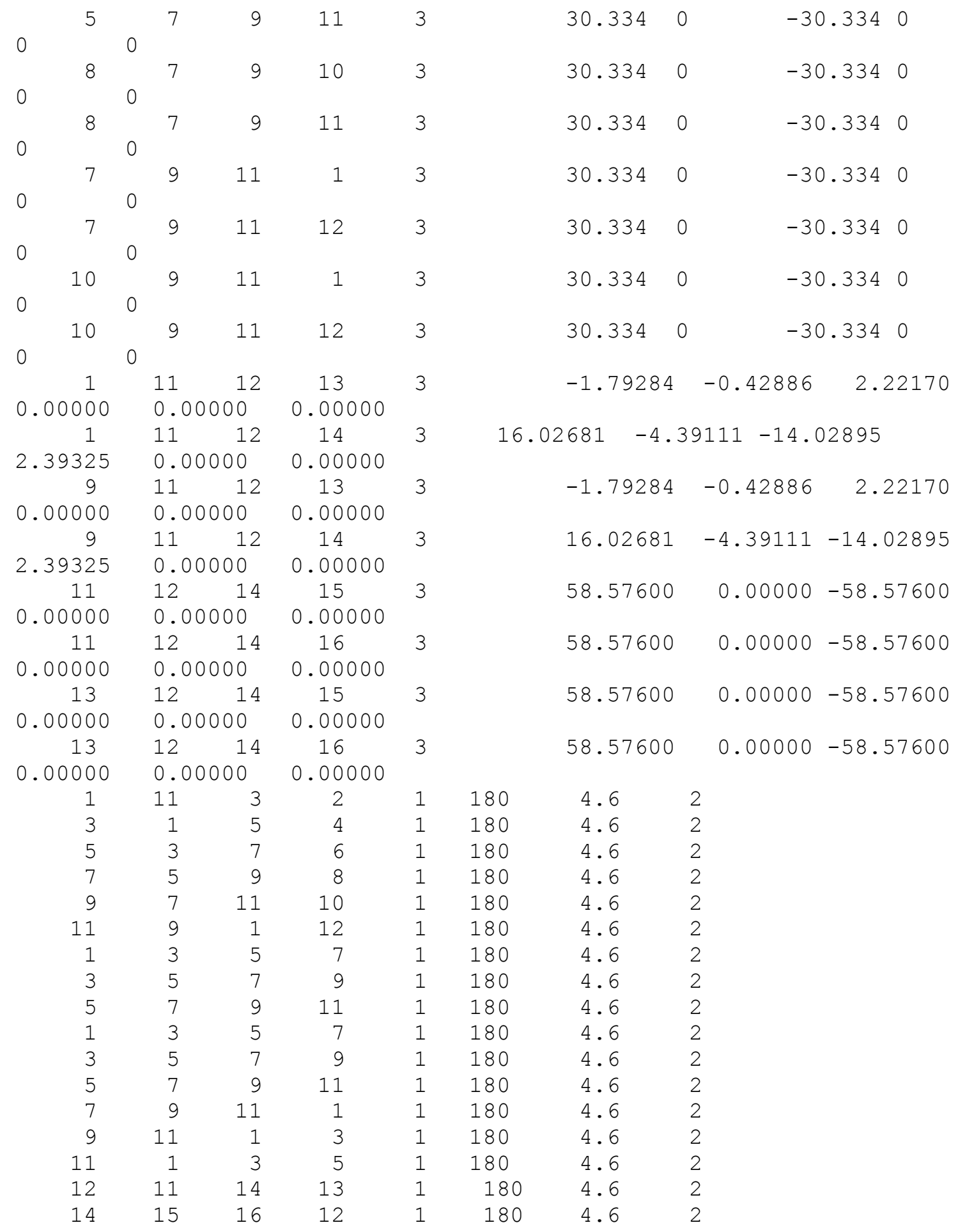

10. toluene

[ moleculetype ]

; Name

TO1

[ atoms]

; nr type typeB chargeB nrexcl 3 


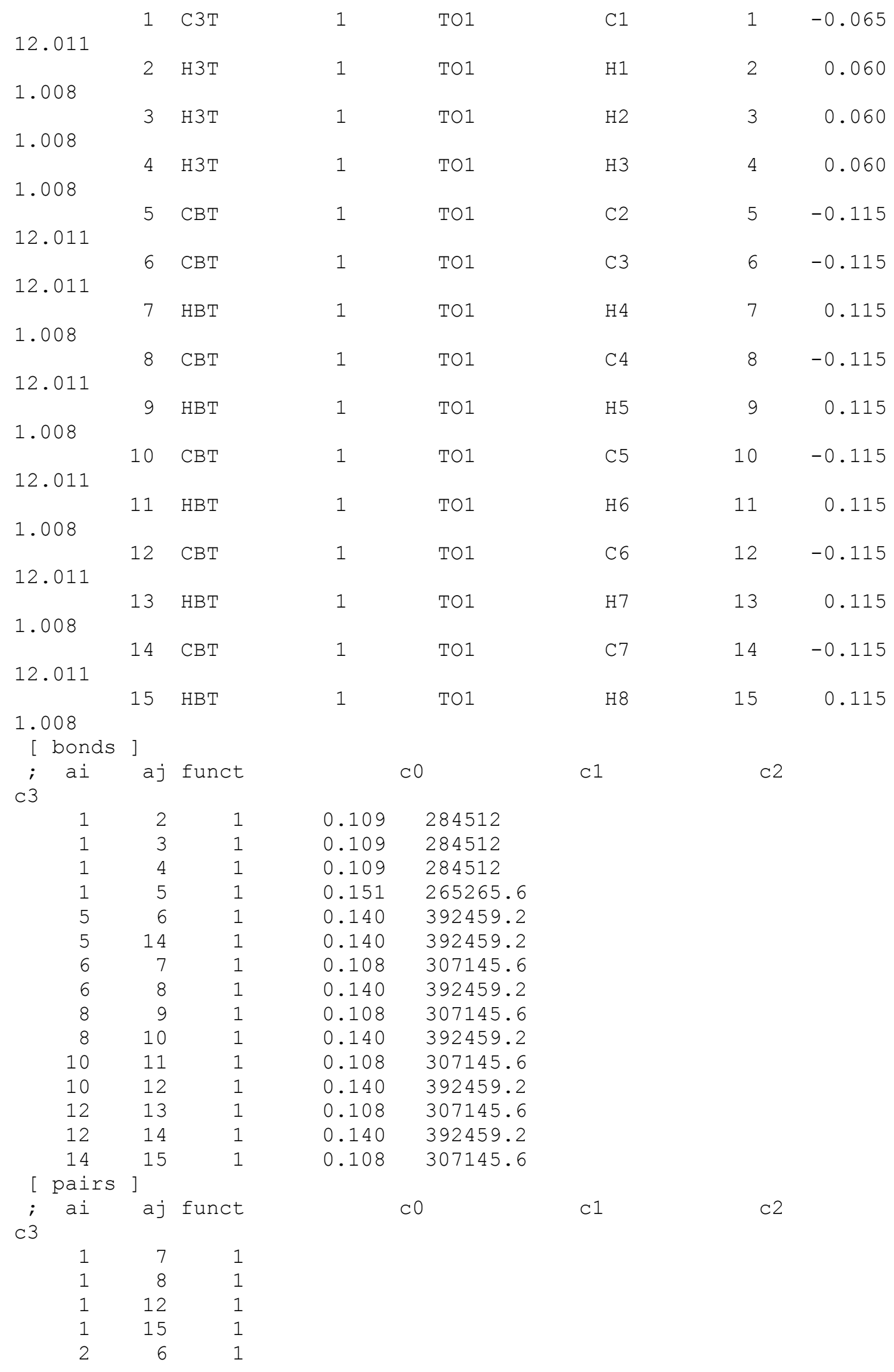




\begin{tabular}{|c|c|c|c|c|c|c|}
\hline 2 & 14 & 1 & & & & \\
\hline 3 & 6 & 1 & & & & \\
\hline 3 & 14 & 1 & & & & \\
\hline 4 & 6 & 1 & & & & \\
\hline 4 & 14 & 1 & & & & \\
\hline 5 & 9 & 1 & & & & \\
\hline 5 & 10 & 1 & & & & \\
\hline 5 & 13 & 1 & & & & \\
\hline 6 & 11 & 1 & & & & \\
\hline 6 & 12 & 1 & & & & \\
\hline 6 & 15 & 1 & & & & \\
\hline 7 & 9 & 1 & & & & \\
\hline 7 & 10 & 1 & & & & \\
\hline 7 & 14 & 1 & & & & \\
\hline 8 & 13 & 1 & & & & \\
\hline 8 & 14 & 1 & & & & \\
\hline 9 & 11 & 1 & & & & \\
\hline 9 & 12 & 1 & & & & \\
\hline 10 & 15 & 1 & & & & \\
\hline 11 & 13 & 1 & & & & \\
\hline 11 & 14 & 1 & & & & \\
\hline 13 & 15 & 1 & & & & \\
\hline [ angle. & ] & & & & & \\
\hline i ai & $a j$ & ak & funct & $\mathrm{co}$ & c1 & \\
\hline 2 & 1 & 3 & 1 & 107.800 & 276.144 & \\
\hline 2 & 1 & 4 & 1 & 107.800 & 276.144 & \\
\hline 2 & 1 & 5 & 1 & 109.500 & 292.880 & \\
\hline 3 & 1 & 4 & 1 & 107.800 & 276.144 & \\
\hline 3 & 1 & 5 & 1 & 109.500 & 292.880 & \\
\hline 4 & 1 & 5 & 1 & 109.500 & 292.880 & \\
\hline 1 & 5 & 6 & 1 & 120.000 & 585.760 & \\
\hline 1 & 5 & 14 & 1 & 120.000 & 585.760 & \\
\hline 6 & 5 & 14 & 1 & 120.000 & 527.184 & \\
\hline 5 & 6 & 7 & 1 & 120.000 & 292.880 & \\
\hline 5 & 6 & 8 & 1 & 120.000 & 527.184 & \\
\hline 7 & 6 & 8 & 1 & 120.000 & 292.880 & \\
\hline 6 & 8 & 9 & 1 & 120.000 & 292.880 & \\
\hline 6 & 8 & 10 & 1 & 120.000 & 527.184 & \\
\hline 9 & 8 & 10 & 1 & 120.000 & 292.880 & \\
\hline 8 & 10 & 11 & 1 & 120.000 & 292.880 & \\
\hline 8 & 10 & 12 & 1 & 120.000 & 527.184 & \\
\hline 11 & 10 & 12 & 1 & 120.000 & 292.880 & \\
\hline 10 & 12 & 13 & 1 & 120.000 & 292.880 & \\
\hline 10 & 12 & 14 & 1 & 120.000 & 527.184 & \\
\hline 13 & 12 & 14 & 1 & 120.000 & 292.880 & \\
\hline 5 & 14 & 12 & 1 & 120.000 & 527.184 & \\
\hline 5 & 14 & 15 & 1 & 120.000 & 292.880 & \\
\hline 12 & 14 & 15 & 1 & 120.000 & 292.880 & \\
\hline [ dihe & als & & & & & \\
\hline$a i$ & $a j$ & $\mathrm{ak}$ & al funct & c & 0 & $\mathrm{c} 1$ \\
\hline c2 & & $c 3$ & C4 & & 5 & \\
\hline 2 & 1 & 5 & 6 & 0.00000 & 0.00000 & 0.00000 \\
\hline 0.00000 & 0.0 & 000 & 0.00000 & & & \\
\hline 2 & 1 & 5 & 14 & 0.00000 & 0.00000 & 0.00000 \\
\hline 0.00000 & 0.0 & 000 & 0.00000 & & & \\
\hline
\end{tabular}




\begin{tabular}{|c|c|c|c|c|c|c|}
\hline 3 & 1 & 6 & 3 & 0.00000 & 0.00000 & 0.00000 \\
\hline 0.00000 & 0.00000 & 0.00000 & & & & \\
\hline 3 & 5 & 14 & 3 & 0.00000 & 0.00000 & 0.00000 \\
\hline 0.00000 & 0.00000 & 0.00000 & & & & \\
\hline 4 & 5 & 6 & 3 & 0.00000 & 0.00000 & 0.00000 \\
\hline .00000 & 0.00000 & 0.00000 & & & & \\
\hline 4 & 5 & 14 & 3 & 0.00000 & 0.00000 & 0.00000 \\
\hline .00000 & 0.00000 & 0.00000 & & & & \\
\hline 1 & 5 & 7 & 3 & 30.33400 & 0.00000 & -30.33400 \\
\hline .00000 & 0.00000 & 0.00000 & & & & \\
\hline 1 & 5 & 8 & 3 & 30.33400 & 0.00000 & -30.33400 \\
\hline 0.00000 & 0.00000 & 0.00000 & & & & \\
\hline 14 & 5 & 7 & 3 & 30.33400 & 0.00000 & -30.33400 \\
\hline 0.00000 & 0.00000 & 0.00000 & & & & \\
\hline 14 & 5 & 8 & 3 & 30.33400 & 0.00000 & -30.33400 \\
\hline 0.00000 & 0.00000 & 0.00000 & & & & \\
\hline 1 & 14 & 12 & 3 & 30.33400 & 0.00000 & -30.33400 \\
\hline 0.00000 & 0.00000 & 0.00000 & & & & \\
\hline 1 & 14 & 15 & 3 & 30.33400 & 0.00000 & -30.33400 \\
\hline 0.00000 & 0.00000 & 0.00000 & & & & \\
\hline 6 & 14 & 12 & 3 & 30.33400 & 0.00000 & -30.33400 \\
\hline 0.00000 & 0.00000 & 0.00000 & & & & \\
\hline 6 & 14 & 15 & 3 & 30.33400 & 0.00000 & -30.33400 \\
\hline 0.00000 & 0.00000 & 0.00000 & & & & \\
\hline 5 & 6 & 9 & 3 & 30.33400 & 0.00000 & -30.33400 \\
\hline 0.00000 & 0.00000 & 0.00000 & & & & \\
\hline 5 & 6 & 10 & 3 & 30.33400 & 0.00000 & -30.33400 \\
\hline 0.00000 & 0.00000 & 0.00000 & & & & \\
\hline 7 & 6 & 9 & 3 & 30.33400 & 0.00000 & -30.33400 \\
\hline 0.00000 & 0.00000 & 0.00000 & & & & \\
\hline 7 & 6 & 10 & 3 & 30.33400 & 0.00000 & -30.33400 \\
\hline 0.00000 & 0.00000 & 0.00000 & & & & \\
\hline 6 & 10 & 11 & 3 & 30.33400 & 0.00000 & -30.33400 \\
\hline 0.00000 & 0.00000 & 0.00000 & & & & \\
\hline $\begin{array}{c}6 \\
0.00000\end{array}$ & $\begin{array}{cc}8 & 10 \\
0.00000\end{array}$ & $\begin{array}{c}12 \\
0.00000\end{array}$ & 3 & 30.33400 & 0.00000 & -30.33400 \\
\hline 9 & 10 & 11 & 3 & 30.33400 & 0.00000 & -30.33400 \\
\hline 0.00000 & 0.00000 & 0.00000 & & & & \\
\hline 9 & 10 & 12 & 3 & 30.33400 & 0.00000 & -30.33400 \\
\hline 0.00000 & 0.00000 & 0.00000 & & & & \\
\hline 8 & 10 & 13 & 3 & 30.33400 & 0.00000 & -30.33400 \\
\hline 0.00000 & 0.00000 & 0.00000 & & & & \\
\hline 8 & 10 & 14 & 3 & 30.33400 & 0.00000 & -30.33400 \\
\hline 0.00000 & 0.00000 & 0.00000 & & & & \\
\hline 11 & 10 & 13 & 3 & 30.33400 & 0.00000 & -30.33400 \\
\hline 0.00000 & 0.00000 & 0.00000 & & & & \\
\hline 11 & 10 & 14 & 3 & 30.33400 & 0.00000 & -30.33400 \\
\hline 0.00000 & 0.00000 & 0.00000 & & & & \\
\hline 10 & 12 & 5 & 3 & 30.33400 & 0.00000 & -30.33400 \\
\hline 0.00000 & 0.00000 & 0.00000 & & & & \\
\hline 10 & 12 & 15 & 3 & 30.33400 & 0.00000 & -30.33400 \\
\hline 0.00000 & 0.00000 & 0.00000 & & & & \\
\hline 13 & 12 & 5 & 3 & 30.33400 & 0.00000 & -30.33400 \\
\hline 0.00000 & 0.00000 & 0.00000 & & & & \\
\hline 13 & 12 & 15 & 3 & 30.33400 & 0.00000 & -30.33400 \\
\hline 0.00000 & 0.00000 & 0.00000 & & & & \\
\hline
\end{tabular}




$\begin{array}{rrrrrrrr}5 & 14 & 6 & 1 & 1 & 180 & 4.6 & 2 \\ 6 & 5 & 8 & 7 & 1 & 180 & 4.6 & 2 \\ 8 & 6 & 10 & 9 & 1 & 180 & 4.6 & 2 \\ 10 & 8 & 12 & 11 & 1 & 180 & 4.6 & 2 \\ 12 & 10 & 14 & 13 & 1 & 180 & 4.6 & 2 \\ 14 & 12 & 5 & 15 & 1 & 180 & 4.6 & 2 \\ 5 & 6 & 8 & 10 & 1 & 180 & 4.6 & 2 \\ 6 & 8 & 10 & 12 & 1 & 180 & 4.6 & 2 \\ 8 & 10 & 12 & 14 & 1 & 180 & 4.6 & 2 \\ 10 & 12 & 14 & 5 & 1 & 180 & 4.6 & 2 \\ 12 & 14 & 5 & 6 & 1 & 180 & 4.6 & 2 \\ 14 & 5 & 6 & 8 & 1 & 180 & 4.6 & 2\end{array}$

11. Urea

[ moleculetype ]

; Name nrexcl

URA 3

[ atoms ]

; nr type resnr resid atom cgnr charge mass total charge

$\begin{array}{lllllll}1-\text { OUrea } & 1 & \text { URA } & 0 & 1 & -0.390 & 15.9994\end{array}$

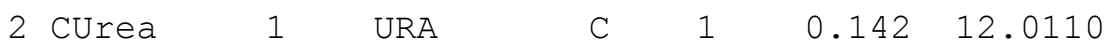

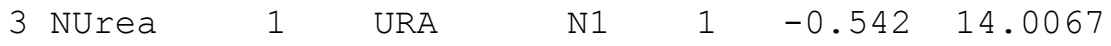

$\begin{array}{llllllll}4 & \mathrm{H} & 1 & \text { URA } & \mathrm{H} 11 & 1 & 0.333 & 1.0080\end{array}$

$\begin{array}{llllllll}5 & \mathrm{H} & 1 & \mathrm{URA} & \mathrm{H} 12 & 1 & 0.333 & 1.0080\end{array}$

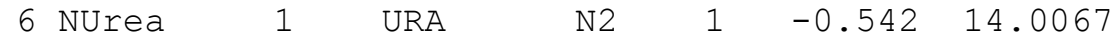

$\begin{array}{llllllll}7 & \mathrm{H} & 1 & \text { URA } & \mathrm{H} 21 & 1 & 0.333 & 1.0080\end{array}$

$\begin{array}{llllllll}8 & \mathrm{H} & 1 & \mathrm{URA} & \mathrm{H} 22 & 1 & 0.333 & 1.0080\end{array}$

; total charge of the molecule: 0.000

[ bonds ]

$\begin{array}{rrccc}\text { i } a i & \text { aj } & \text { funct } & c 0 & c 1 \\ 1 & 2 & 2 & 0.1265 & 1.3100 e+07 \\ 2 & 3 & 2 & 0.1350 & 1.0300 e+07 \\ 2 & 6 & 2 & 0.1350 & 1.0300 e+07 \\ 3 & 4 & 2 & 0.1000 & 1.8700 e+07 \\ 3 & 5 & 2 & 0.1000 & 1.8700 e+07 \\ 6 & 7 & 2 & 0.1000 & 1.8700 e+07 \\ 6 & 8 & 2 & 0.1000 & 1.8700 e+07\end{array}$

[ pairs ]

; ai aj funct; all 1-4 pairs but the ones excluded in GROMOS itp

[ angles ]

; ai aj ak funct angle fc

$\begin{array}{llllll}1 & 2 & 3 & 2 & 121.40 & 690.00\end{array}$

$\begin{array}{llllll}1 & 2 & 6 & 2 & 121.40 & 690.00\end{array}$

$\begin{array}{llllll}3 & 2 & 6 & 2 & 117.20 & 636.00\end{array}$

$\begin{array}{llllll}2 & 3 & 4 & 2 & 120.00 & 390.00\end{array}$

$\begin{array}{llllll}2 & 3 & 5 & 2 & 120.00 & 390.00\end{array}$

$\begin{array}{llllll}4 & 3 & 5 & 2 & 120.00 & 445.00\end{array}$

$\begin{array}{llllll}2 & 6 & 7 & 2 & 120.00 & 390.00\end{array}$

$\begin{array}{llllll}2 & 6 & 8 & 2 & 120.00 & 390.00\end{array}$

[ dihedrals ]

; GROMOS improper dihedrals

; ai aj ak al funct angle fc

$\begin{array}{lllllll}2 & 3 & 6 & 1 & 2 & 0.00 & 167.36\end{array}$ 


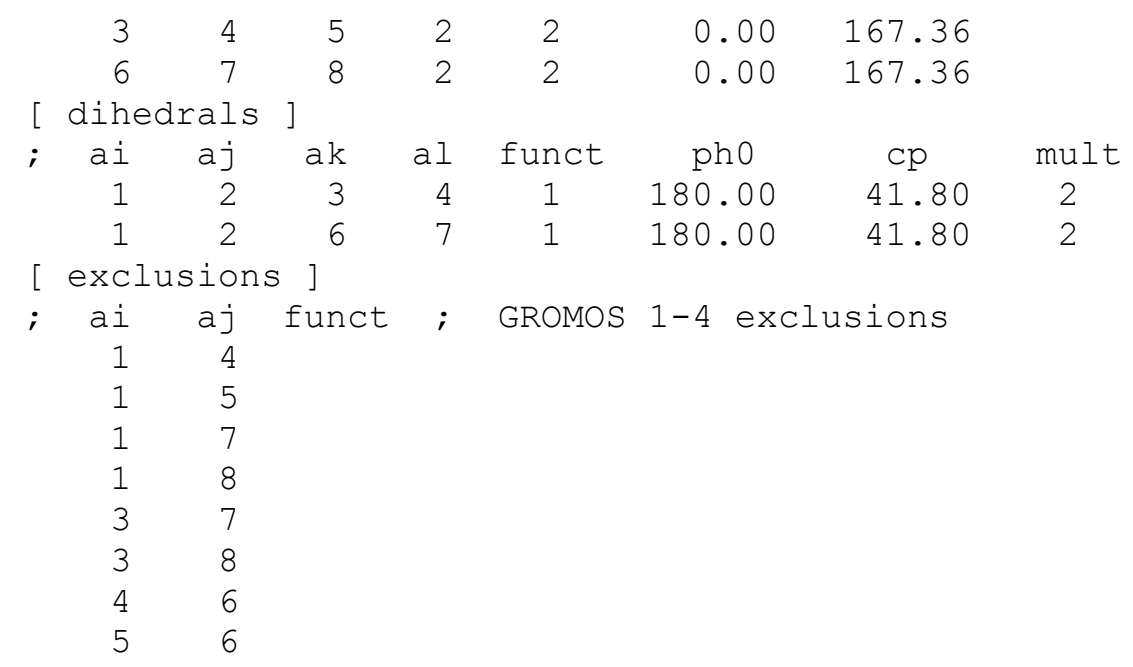

